\title{
On the Early Development of Dispersion in Flow through a Tube with Wall Reactions
}

\author{
M. W. Lau, and C. O. Ng
}

\begin{abstract}
This is a study on numerical simulation of the convection-diffusion transport of a chemical species in steady flow through a small-diameter tube, which is lined with a very thin layer made up of retentive and absorptive materials. The species may be subject to a first-order kinetic reversible phase exchange with the wall material and irreversible absorption into the tube wall. Owing to the velocity shear across the tube section, the chemical species may spread out axially along the tube at a rate much larger than that given by the molecular diffusion; this process is known as dispersion. While the long-time dispersion behavior, well described by the Taylor model, has been extensively studied in the literature, the early development of the dispersion process is by contrast much less investigated. By early development, that means a span of time, after the release of the chemical into the flow, that is shorter than or comparable to the diffusion time scale across the tube section. To understand the early development of the dispersion, the governing equations along with the reactive boundary conditions are solved numerically using the Flux Corrected Transport Algorithm (FCTA). The computation has enabled us to investigate the combined effects on the early development of the dispersion coefficient due to the reversible and irreversible wall reactions. One of the results is shown that the dispersion coefficient may approach its steady-state limit in a short time under the following conditions: (i) a high value of Damkohler number (say $D a \geq 10$ ); (ii) a small but non-zero value of absorption rate (say $\Gamma^{*} \leq 0.5$ ).
\end{abstract}

Keywords-Dispersion coefficient, early development of dispersion, FCTA, wall reactions.

\section{INTRODUCTION}

$\mathrm{D}$ ISPERSION in laminar flow through a tube has been extensively studied for the past five decades, since the classical works by Taylor [1] and Aris [2]. These studies have largely been focused on the long-time dispersion phenomena, which are well described by the Taylor model. In contrast, studies on the short-time dispersion behaviors have been very limited [3, 4]. By short-time dispersion, it means that the dispersion has been developing only for a time scale that is shorter than or comparable to the diffusion time scale across the tube section. The present study on the early-phase dispersion is motivated by its applications to (i) transport in human arteries, which are typically short tubes, over which the travel time may

Manuscript received May 4, 2007. This work was supported by the Research Grants Council of the Hong Kong Special Administrative Region, China, through Project No. HKU 7192/04E.

M. W. Lau is with the Department of Mechanical Engineering, The University of Hong Kong, Pokfulam Road, Hong Kong, China (phone: 852-92626064; fax: 852-28585415; e-mail: tobylau@hkusua.hku.hk).

C. O. Ng is with the Department of Mechanical Engineering, The University of Hong Kong, Pokfulam Road, Hong Kong, China (e-mail: cong@hku.hk). not be long enough for the long-time behaviors to materialize; (ii) chromatographic separation, which are concerned with the multiple peaks arising from the non-Gaussian axial concentration distribution during an early phase of transport; and (iii) the specification of a proper initial condition for a long-time dispersion problem. Despite its practical significance, dispersion under the combined effects of kinetic reversible phase exchange and irreversible absorption has also not been fully investigated in the literature.

The present study aims to investigate the short-time transient dispersion behavior as a function of the wall reaction parameters. The classical techniques used to find the long-time dispersion, which can be studied analytically by a one-dimensional transport model in terms of the section-averaged concentration, is not suitable for the present study. It is because the radial variation of the concentration is as important as the axial variation during the early development. The transport in an early phase is essentially two dimensional, thereby making the problem not amenable to the analytical approach as can be applied to the long-time one-dimensional problem. Therefore, the short-time problem could be solved by a well-devised numerical method. It is also of practical interest to look into how the dispersion will develop from the initial stage to its long-time Taylor-model limit. The central questions to be addressed are as follows. How much time does it take to approach the long-time limit? How does the axial concentration distribution evolve from non-Gaussian initially to Gaussian finally? To what extent are these two aspects of asymptotic matching affected by the kinetic reversible and irreversible wall reactions? All these points raised above have formed the motivation of this study. In the present study, numerical simulations are performed with a view to look into the early phases of dispersion due to axial convection and radial diffusion in steady flow along a circular tube, subject to the boundary conditions of kinetic reversible phase exchange and irreversible absorption on the wall.

\section{MATHEMATICAL MODEL}

For fully-developed steady laminar flow through a straight tube of circular section, the equation of transport of a species with no chemical reaction in the fluid is

$$
\frac{\partial C}{\partial t}+u \frac{\partial C}{\partial z}=D \frac{\partial^{2} C}{\partial z^{2}}+\frac{D}{r} \frac{\partial}{\partial r}\left(r \frac{\partial C}{\partial r}\right), \quad 0<r<a
$$


where $r$ is the radial coordinate, $Z$ is the axial coordinate, $a$ is the radius of the tube, $D$ is the molecular diffusion coefficient of the chemical in the fluid, and $u$ is the laminar velocity profile given by $u=u_{o}\left(1-r^{2} / a^{2}\right)$. Here $u_{o}$ is the velocity of the flow at the center of the tube. Then, (1) becomes

$$
\frac{\partial C}{\partial t}+u_{o}\left(1-\frac{r^{2}}{a^{2}}\right) \frac{\partial C}{\partial z}=D \frac{\partial^{2} C}{\partial z^{2}}+\frac{D}{r} \frac{\partial}{\partial r}\left(r \frac{\partial C}{\partial r}\right)
$$

Along with the transport equation, the boundary conditions in the present dispersion problem are

$$
\begin{gathered}
\frac{\partial C}{\partial r}=0, \quad \text { at } r=0 \\
-D \frac{\partial C}{\partial r}-\Gamma C=\frac{\partial C_{S}}{\partial t}=k\left(\alpha C-C_{S}\right), \quad \text { at } r=a
\end{gathered}
$$

Here, $C(z, r, t)$ is the concentration (mass of species dissolved per bulk volume of fluid) of the mobile phase, $C_{S}(z, t)$ is the concentration (mass of species retained per surface area of wall) of the immobile phase, $\Gamma, k$ and $\alpha$ are the irreversible absorption rate, the reversible reaction rate and the partition coefficient, respectively. See Ng [5] for details about these boundary conditions.

Let us introduce the following normalized variables (distinguished by an asterisk):

$$
\begin{aligned}
& C^{*}=\frac{C}{C_{0}}, C_{s}^{*}=\frac{C_{s}}{a C_{0}}, r^{*}=\frac{r}{a}, z^{*}=\frac{z}{a^{2} u_{0} / D}, \\
& t^{*}=\frac{t}{a^{2} / D}, \alpha^{*}=\frac{\alpha}{a}, \Gamma^{*}=\frac{\Gamma a}{D}, D a=\frac{k a^{2}}{D}, \\
& P e=\frac{u_{0} a}{D}
\end{aligned}
$$

by which the transport equation and boundary conditions (2)-(4) can be written as follows:

$$
\begin{gathered}
\frac{\partial C^{*}}{\partial t^{*}}+\left(1-r^{* 2}\right) \frac{\partial C^{*}}{\partial z^{*}}=\frac{1}{P e^{2}} \frac{\partial^{2} C^{*}}{\partial z^{* 2}} \\
+\frac{1}{r^{*}} \frac{\partial}{\partial r^{*}}\left(r^{*} \frac{\partial C^{*}}{\partial r^{*}}\right), \quad 0<r<1 \\
\frac{\partial C^{*}}{\partial r^{*}}=0, \quad \text { at } r=0
\end{gathered}
$$

$-\frac{\partial C^{*}}{\partial r^{*}}-\Gamma^{*} C^{*}=\frac{\partial C_{S}^{*}}{\partial t^{*}}=\operatorname{Da}\left(\alpha^{*} C^{*}-C_{S}^{*}\right), \quad$ at $r=1$

The computation gives solutions for a two-dimensional concentration field $C^{*}\left(z^{*}, r^{*}, t^{*}\right)$. It is also of interest to express the results in terms of some averaged concentrations. The most widely used one is

$$
\bar{C}^{*}=2 \int_{0}^{1} C^{*} r^{*} d r^{*}
$$

where $\bar{C}^{*}$ is the area average concentration.

The dispersion coefficient $D c$, which varies with time, is given by half the rate of change of the variance of the distribution, $\sigma^{2}$ :

$$
\sigma^{2}(t)=\frac{\mu_{2}}{\mu_{0}}-\frac{\mu_{1}^{2}}{\mu_{0}^{2}} ; \quad D c(t)=\frac{1}{2} \frac{d \sigma^{2}}{d t}
$$

where $\mu_{n}$ is the $n$th axial integral moment of $C^{*}$ :

$\mu_{n}=\int_{-\infty}^{\infty} z^{* n} C^{*} d z^{*}(n=0,1,2, \cdots)$

\section{NUMERICAL ALGORITHM}

In the simulations, the chemical is assumed to be released initially in the form of a narrow slug into the flow. It demands great care in order to obtain accurate numerical solutions for the transport of an impulse input when convection dominates. Otherwise, for high Peclet number, a sharp axial concentration gradient may result in either numerical smearing, which confuses the physical dispersion, or numerical oscillation, which corrupts the entire solution. In this regard, the FCTA is adopted to solve numerically the transport equation for the present problem. The advantages of the FCTA over other well-known numerical methods (e.g., Lax-Wendroff and Crank-Nicholson) for the convection-dominated transport problems have been demonstrated by Boris and collaborators [6 - 9]. Essentially, FCTA consists of two major stages: a convective-diffusive stage (Stage I) followed by an antidiffusive or corrective stage (Stage II). Both stages are conservative and maintain positivity. The two steps enable FCTA to treat strong gradients and shocks without the usual dispersive ripples. 


\section{RESUlts AND Discussion}

All of the results presented here were obtained with the following finite difference parameters: $\Delta t^{*}=0.0001$, $\Delta z^{*}=0.001, \Delta r^{*}=0.0333$ and $P e=1000$. Sufficiently small spatial discretizations have been utilized in order to keep the accuracy of the results. The initial slug length was fixed at $z_{S}^{*}=8\left(\Delta z^{*}\right)$. To investigate the coupled effects of the reversible phase exchange and irreversible absorption, simulations have been generated for five cases of different parameters as listed in Table I. These parameters include $\alpha^{*}$, $\mathrm{Da}$ and $\Gamma^{*}$. While $\alpha^{*}$ is the ratio of the chemical species distributed between the mobile phase and immobile phase, the Damkohler number $D a$ is the ratio of the phase exchange rate to the diffusion rate and the $\Gamma^{*}$ is the absorption rate. In particular, case 1 is an inert case where $\alpha^{*}=0$ and $\Gamma^{*}=0$. Cases 2-4 are to show the effects of $D a$, while cases 3 and 5 are to show the effects of $\Gamma^{*}$.

Mean breakthrough curves based on the area-average concentration for the five cases are presented in Figs. 1- 4 for four axial locations $\left(z^{*}=0.05, Z^{*}=0.1, Z^{*}=0.15\right.$, and $\left.Z^{*}=0.25\right)$. In Fig. 1, double peaks show up in cases 1-3, which are caused by the strong spatial segregation of convection and diffusion regimes. The first peak is the predominance of convection in the central region of the tube. Meanwhile, the second peak is due to the development of a stronger diffusion influence near the tube wall. However, the phenomenon of double-peak does not show up in cases 4 and 5 . This implies that the second peak arising from the diffusion regime will be suppressed by either a high value of the Damkohler number, by comparing cases $1-4$, or a relative high absorption rate, by comparing cases 3 and 5 . In Fig. 2, the second peak, which is caused by the diffusion, becomes the dominant feature of the mean breakthrough curve in cases 1-3. Similarly, the second peak does not show up in cases 4 and 5 due to relatively fast retentive or absorptive effects. Also, it is found that there is a long tail in case 4. It is because some chemical species at the immobile phase will be dispersed back to the mobile phase owing to the fast phase exchange rate. Therefore, a steady state can be approached in a short time, if $D a$ is high enough (say $D a \geq 10$ ). In Fig. 3, the diffusional peak is broadened and has become the main feature of the mean breakthrough curve with the last residue of a convection regime appearing as a small shoulder on the left of the curve. In addition, area under the distribution is diminished in cases 4 and 5, corresponding to a fast phase exchange rate and strong absorption, respectively. In Fig. 4, the mean breakthrough curves develop toward being symmetrical about the peak, on approaching a Gaussian distribution in cases 1-2. Meanwhile, despite the fast retentive exchange, a long tail is still seen in case 4 . Also, owing to the large absorptive effect, the area under the distribution in case 5 becomes smaller compared with that in case 3. It happens as the chemical species keeps on being depleted by wall absorption.
TABLE I

PARAMETERS OF $\alpha *, D a$ AND $\Gamma *$ FOR FIVE CASES OF THE SIMULATIONS, WHERE CASES 2-4 CORRESPOND TO THE INCREASE OF PHASE EXCHANGE RATE AND CASES 3 AND 5 CORRESPOND TO THE INCREASE OF ABSORPTION RATE

\begin{tabular}{cccc}
\hline \hline Case & $\alpha^{*}$ & $D a$ & $\Gamma^{*}$ \\
\hline 1 & 0 & 0 & 0 \\
2 & 0.5 & 0.1 & 0.1 \\
3 & 0.5 & 1 & 0.1 \\
4 & 0.5 & 10 & 0.1 \\
5 & 0.5 & 1 & 1 \\
\hline \hline
\end{tabular}

Snapshots of the mobile-phase concentration distributions are plotted in Figs. 5-8 to illustrate the dispersion behavior of the chemical species at four instants of time $\left(t^{*}=0.05\right.$, $t^{*}=0.15, t^{*}=0.3$, and $\left.t^{*}=1.5\right)$. Fig. 5 shows that the area under the distribution curve is markedly diminished for both cases 4 and 5, corresponding to fast phase exchange and absorption rates, respectively. Fig. 6 shows a larger spread of the distribution in both cases arising from the interaction of convection with radial diffusion. Similarly, the diminishing of area under the distribution curves is clearly seen in cases 4 and 5 as mass is taken away from the flow by either the wall retention or wall absorption. In particular, in case 4 corresponding to very fast phase exchange, it can be observed that an additional peak is exhibited at $z^{*}=0.01$. This is a result of releasing mass, which has been stored as an immobile phase on the wall, back to the flow. Fig. 7 shows that, for cases 1-3 and 5, the distribution curves are single-peaked. However, double peaks are clearly displayed for case 4 , as a result of fast phase exchange. In Fig. 8, it is clear that the skewness is very small in case 1, supporting that the Gaussian form is readily developed in this case. In case 4, the leading peak continues to broaden to become the dominant feature of the distribution without a shoulder. Owing to the fast phase exchange in case 4, it approaches a fully-developed state quickly. On the other hand, for case 3 corresponding to slow phase exchange, a shoulder and even a second peak at $Z^{*}=0.3$ are exhibited owing to the retentive effect. This is because a longer time is required to approach the fully-developed state if the phase exchange is so slow (say $D a \leq 1$ ) that it becomes rate-determining. In addition, it is clear that the area under the concentration distribution curve in case 5 is smaller than that in case 3. This follows from the fact that for a very high wall absorption rate (say $\Gamma^{*} \geq 10$ ), the long-time dispersion will not be attained before virtually all mass is taken away from the system, and hence it may not have any practical significance. 


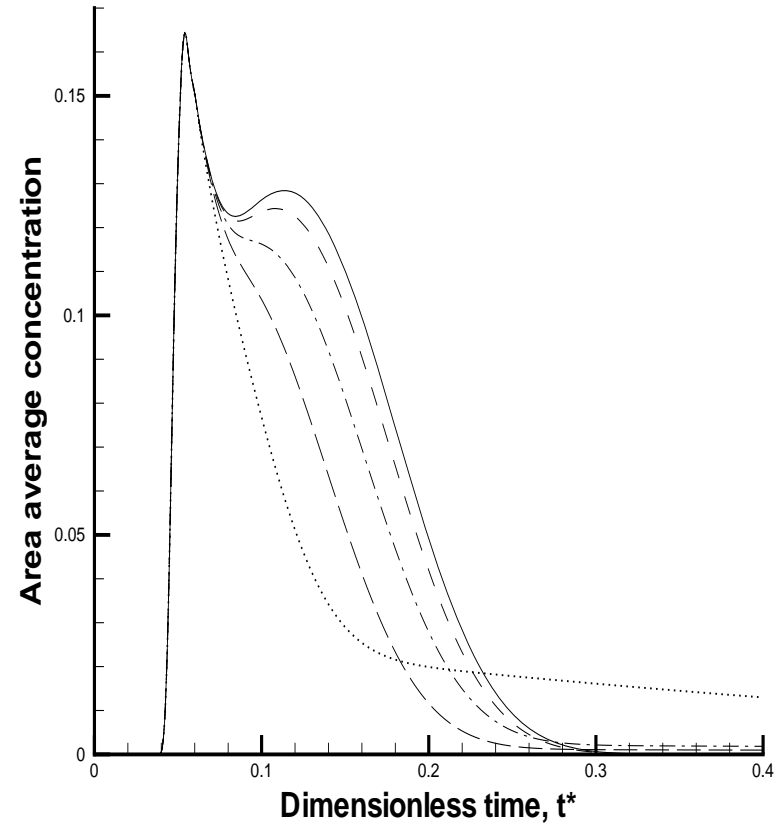

Fig. 1 Area-average concentration profiles of the mobile phase at

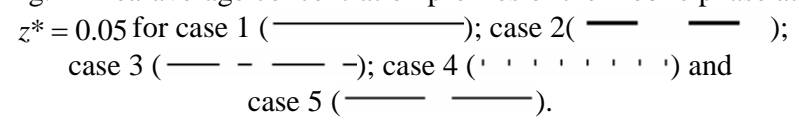

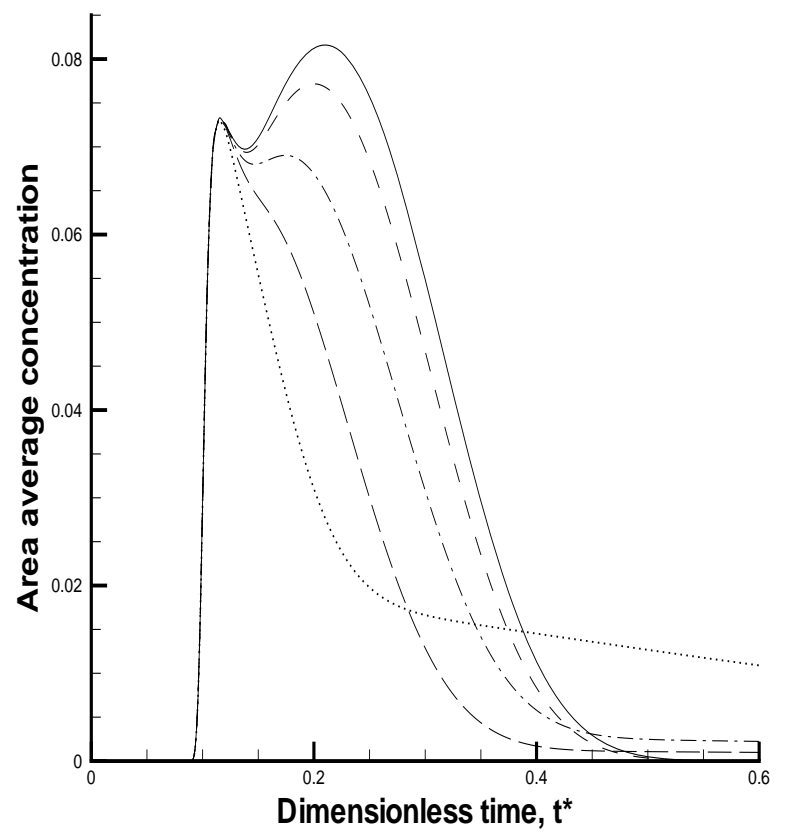

Fig. 2 Area-average concentration profiles of the mobile phase at $Z^{*}=0.1$ for different cases as in Fig. 1

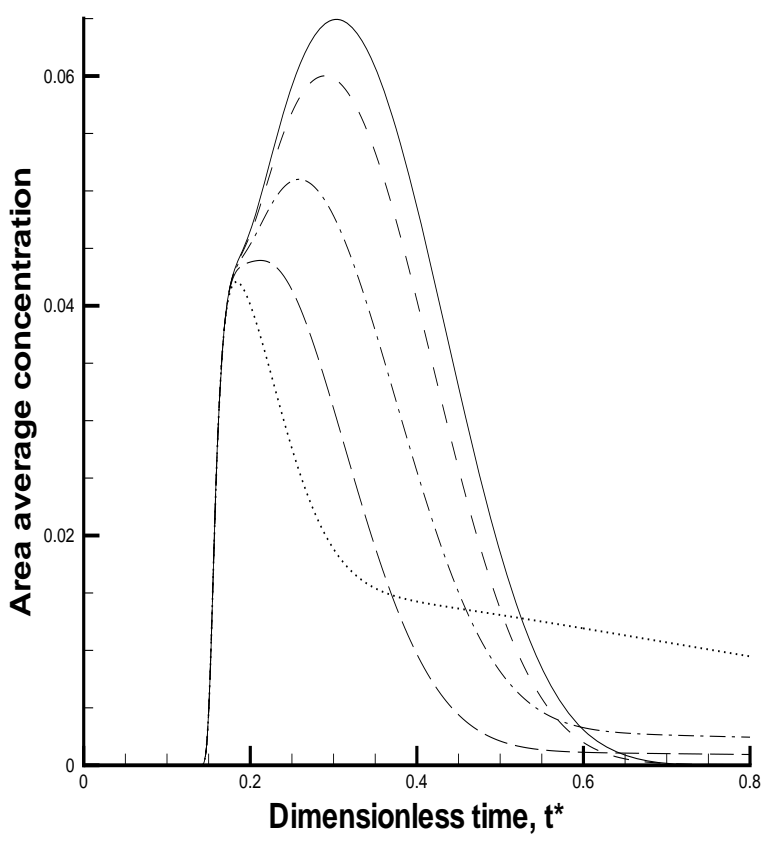

Fig. 3 Area-average concentration profiles of the mobile phase at $z^{*}=0.15$ for different cases as in Fig. 1

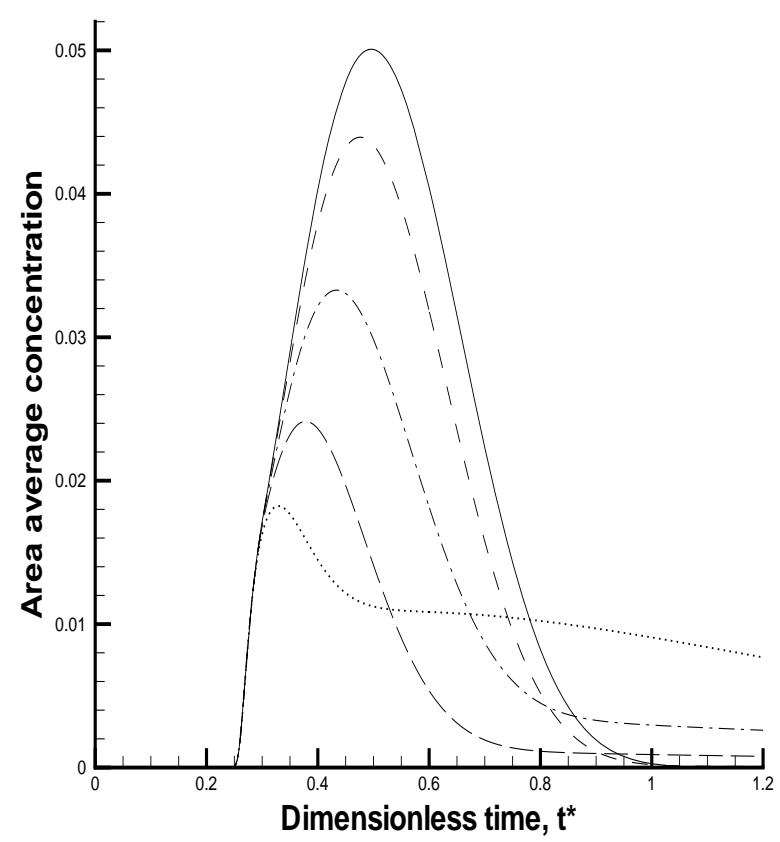

Fig. 4 Area-average concentration profiles of the mobile phase at $z^{*}=0.25$ for different cases as in Fig. 1 


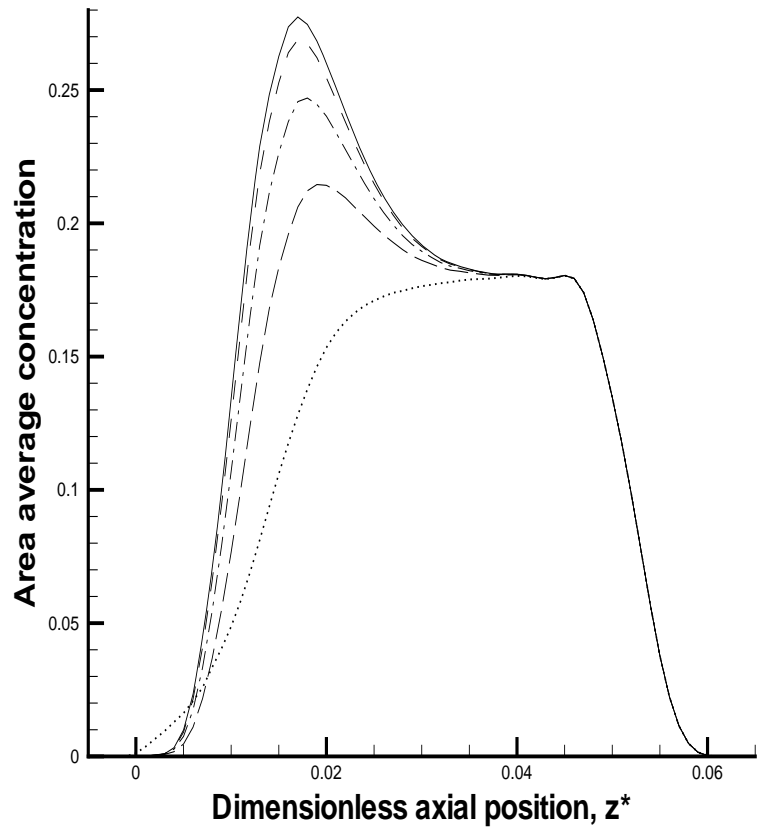

Fig. 5 Snapshot of the concentration distribution of the mobile phase at $t^{*}=0.05$ for different cases as in Fig. 1

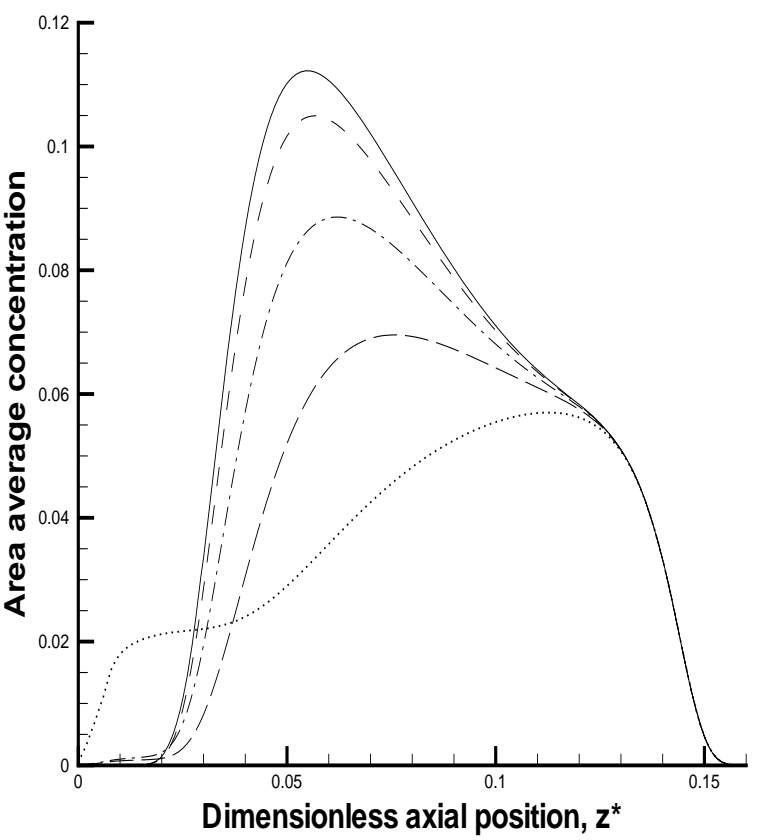

Fig. 6 Snapshot of the concentration distribution of the mobile phase at $t^{*}=0.15$ for different cases as in Fig. 1

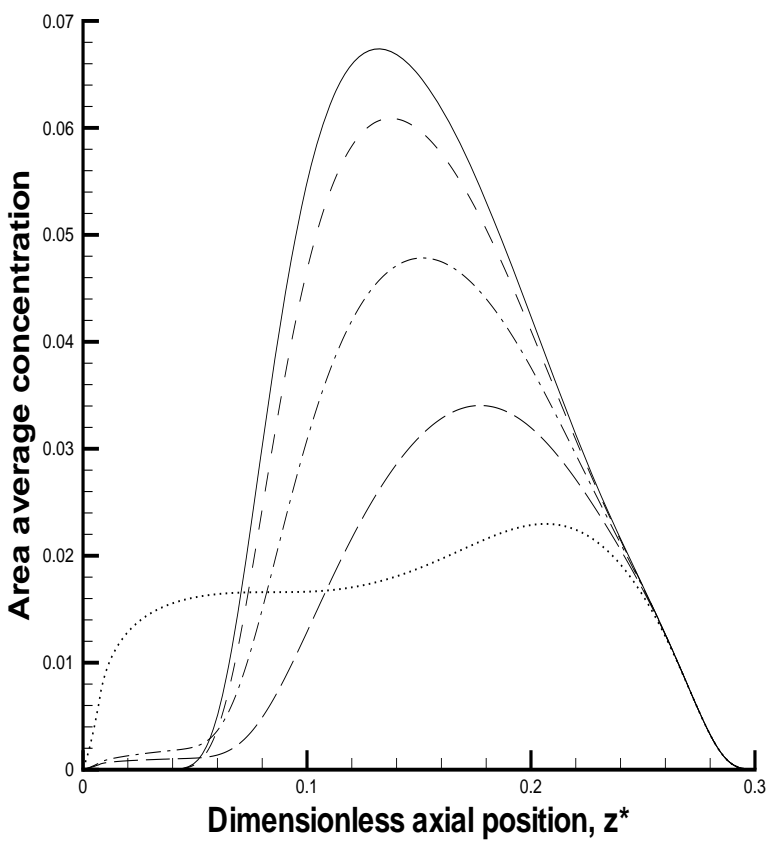

Fig. 7 Snapshot of the concentration distribution of the mobile phase at $t^{*}=0.3$ for different cases as in Fig. 1

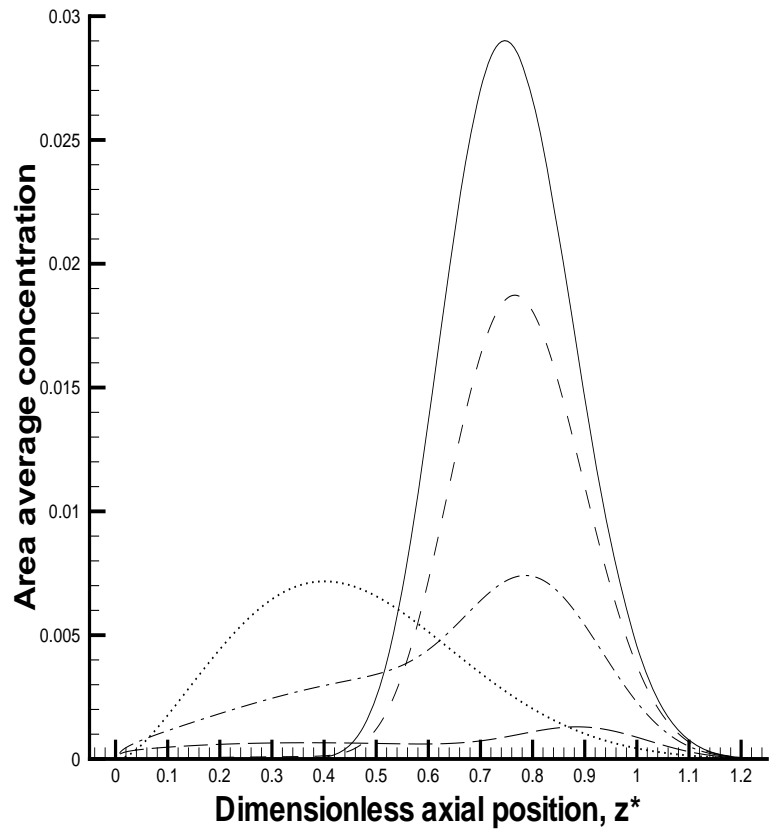

Fig. 8 Snapshot of the concentration distribution of the mobile phase at $t^{*}=1.5$ for different cases as in Fig. 1 
Fig. 9 shows the early-time development of the dispersion coefficient for the five cases. The following observations can be made. First, the dispersion coefficient tends to a steady value very quickly, after $t^{*}=0.2$, for case 1 without reactions. Second, for cases 2 and 3 corresponding to very slow phase exchange rates, their dispersion coefficients will not attain the steady-state values in a short time owing to the slow retentive effect. For case 4 corresponding to a fast phase exchange rate, the dispersion coefficient rises to a local peak before it decreases to approach a steady value by $t^{*}=1$. This implies that the dispersion coefficient can approach a steady-state limit in a short time, if the value of the $D a$ is high enough (say $D a \geq 10$ ). Third, by comparing cases 3 and 5 , it is clear that the dispersion coefficient in case 5 can be momentarily higher but eventually smaller than that in case 3. Observably, an increase in the dispersion coefficient in the absorptive case does not necessarily correspond to a larger spread of the distribution, but instead it can be caused by a diminishing area under the distribution curve as mass keeps on being depleted by wall absorption. Therefore, as remarked above, for too large a wall absorption rate, the long-time limit of the dispersion coefficient may not have practical significance. Long before this limit is attained, all the mass will have been removed from the system. This point deserves further investigation.

\section{CONCLUding REMARKS}

Applying the FCTA, numerical simulations have been performed for the early time-development of mass transport, by convection and diffusion, of a chemical species in steady flow through a tube under the influences of reversible and irreversible wall reactions. The mean breakthrough curves, snapshots of the axial distribution and time evolution of the dispersion coefficient as a function of the reaction parameters have been investigated. The following summary can be made. First, the second peak of the diffusion regime will be affected and restricted by a high value of the Damkohler number and a high absorption rate. Second, it requires a longer time to approach a long-time dispersion if the phase exchange rate is so slow (say $D a \leq 1$ ). Third, under the following conditions, the dispersion coefficient may approach its steady-state limit in a short time: (i) a high value of Damkohler number (say $D a \geq 10$ ); (ii) a small but non-zero value of absorption rate (say $\Gamma^{*} \leq 0.5$ ). These results will help us further understand the dispersion in its early development.

\section{ACKNOWLEDGMENTS}

The work was supported by the Research Grants Council of the Hong Kong Special Administrative Region, China, through Project No. HKU 7192/04E, and also by the University of Hong Kong through the Seed Funding Programme for Basic Research A/C 10207452. This study constitutes part of the work performed by the first author for her M.Phil. thesis under the supervision of the second author at the University of Hong Kong.

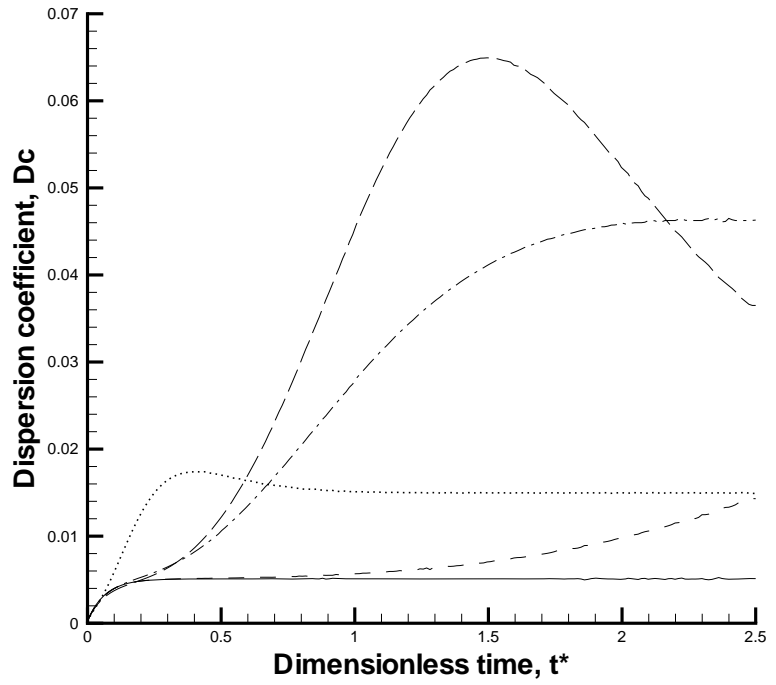

Fig. 9 Dispersion coefficient versus dimensionless time for different cases as in Fig. 1

\section{REFERENCES}

[1] G.I. Taylor, "Dispersion of soluble matter in solvent flowing slowly through a tube,” Proc. R. Soc. Lond. A, vol. 219, pp.186-203, 1953.

[2] R. Aris, "On the dispersion of a solute in a fluid flowing through a tube," Proc. R. Soc. Lond. A, vol. 235, pp.67-77, 1956.

[3] M.J. Lighthill, "Initial development of diffusion in Poiseuille flow," $J$. Inst. Math. Appl., vol. 2, pp.97-108, 1956.

[4] P.C. Chatwin, "The initial dispersion of contaminant in Poiseuille flow and the smoothing of the snout,” J. Fluid Mech., vol. 77, pp.593-602, 1976.

[5] C.O. Ng, "Dispersion in steady and oscillatory flows through a tube with reversible and irreversible wall reactions," Proc. R. Soc. A, vol. 462, pp.481-515, 2006.

[6] J.P. Boris and D.L. Book, "Flux-corrected transport 1. Shasta, a fluid transport algorithm that works,” J. Comp. Phys., vol. 11, pp.38-69, 1973.

[7] D.L. Book, J.P. Boris and K. Hain, "Flux-corrected transport 2. Generalizations of method,” J. Comp. Phys., vol. 18, pp.248-283, 1975.

[8] J.P. Boris and D.L. Book, "Flux-corrected transport 3. Minimal-error fct algorithms,” J. Comp. Phys., vol. 20, pp.397-431, 1976.

[9] J.P. Boris, A.M. Landsberg, E.S. Oran and J.H. Gardner, "LCPFCT-a flux-corrected transport algorithm for solving generalized continuity equations”, Report NRL/MR/6410-93-7192, National Research Laboratory, Washington DC, USA, 1993. 